Original research

\title{
An education intervention care bundle to improve hospitalised patients' pressure injury prevention knowledge: a before and after study
}

\section{Deakin J, Gillespie BM, Chaboyer W, Nieuwenhoven P and Latimer S}

Keywords patient participation, pressure ulcers, implementation, technology, patient teaching

For referencing Deakin $\mathrm{J}$ et al. An education intervention care bundle to improve hospitalised patients' pressure injury prevention knowledge: a before and after study. Wound Practice and Research 2020; 28(4):154-162.

DOI https://doi.org/10.33235/wpr.28.4.154-162

\section{Jodie Deakin*}

GCEF, BN, RN

Clinical Research Nurse, School of Nursing and

Midwifery, Griffith University, Gold Coast, QLD,

Australia

Nursing and Midwifery Education and Research

Unit, Gold Coast Hospital and Health Service,

Southport, QLD, Australia

Email j.deakin@griffith.edu.au

\section{Brigid M Gillespie}

PhD, RN, FACORN

Professor of Patient Safety, School of Nursing and

Midwifery, Griffith University, Gold Coast, QLD,

Australia

Menzies Health Institute Queensland, QLD,

Australia

Nursing and Midwifery Education and Research

Unit, Gold Coast Hospital and Health Service,

Southport, QLD, Australia

\section{Wendy Chaboyer}

PhD, ACCCN, FAAN, Phi Delta-at-Large Chapter

STTI

Deputy Head of School (Research), School of

Nursing and Midwifery, Griffith University, Gold

Coast, QLD, Australia

Menzies Health Institute Queensland, QLD,

Australia

\section{Paul Nieuwenhoven}

Director of Nursing, Surgical, Anaesthetics and

Procedural Services, Gold Coast Hospital and

Health Service, Southport, QLD, Australia

\section{Sharon Latimer}

PhD, MAP, MN, Grad Dip L\&T, BN, RN

Senior Research Fellow, School of Nursing and

Midwifery, Griffith University, QLD, Australia

Menzies Health Institute Queensland, QLD,

Australia

Nursing and Midwifery Education and Research

Unit, Gold Coast Hospital and Health Service,

Southport, QLD, Australia

* Corresponding author

\section{Abstract}

Aim To measure the relationship between patient participation in pressure injury prevention (PIP) both before and after implementing a patient-centred pressure injury prevention care bundle (PPIPCB).

Methods This before and after intervention study used surveys to measure adult medical patients' participation in their PIP care and satisfaction with the PPIPCB across three medical inpatient units in an Australian tertiary hospital. Descriptive statistics described the sample and patient intervention satisfaction. A paired samples t-test measured changes in the before and after survey scale scores.

Results Eighty patients, with an average age of 67.2 years $(\mathrm{SD}=18.3)$, were recruited during November and December 2019. There were statistically significant increases in total mean scores for the patient participation in pressure injury prevention (PPPIP) scale after the intervention (before: mean=18.6, $S D=2.8$; after: mean=22.5, $S D=1.9$ ). Significant improvements in patients' self-reported knowledge of 
pressure injury $(\mathrm{PI})$ risk and perceived levels of participation in their PI care were reported after receiving the intervention. A total of $98.8 \%$ of participants agree or strongly agree that they were satisfied with the intervention as delivered.

Conclusion Engaging patients is one way to increase their participation in PIP. Wider clinical adoption appears warranted, with nurses the key to the successful implementation.

\section{Impact}

What is already known?

- Hospital-acquired pressure injuries (HAPIs) are a serious adverse event that can result in physical and psychological consequences, increased morbidity and mortality.

- Well-designed education care bundles can reduce adverse events in hospitalised patients.

What does this implementation project contribute?

- Implementing an evidence-based patient-centred education care bundle intervention improves patients' knowledge of Pls and their prevention.

- Providing clinicians with PIP teaching resources facilitates patient education and their participation in this aspect of their care.

\section{Background}

Pressure injuries $(\mathrm{PI})$ or pressure ulcers result from continuous and/or unrelieved pressure, friction or shear causing damage to the underlying tissue and skin 1 . Pls occurring in the acute care setting are known as a hospital-acquired pressure injury (HAPI) ${ }^{2,3}$. Despite proactive interventions ${ }^{1}$, global HAPI rates have remained relatively unchanged since 2010 , with a reported combined PI pooled prevalence of $12.8 \%{ }^{4}$, whilst an $8.4 \%$ pooled prevalence HAPI rate was found amongst $1,893,593$ hospitalised adult patients in a 2020 metaanalysis ${ }^{4}$.

HAPIs are largely preventable adverse events ${ }^{5}$ which can result in increased hospital length of stay (HLOS) of up to 20 days $^{6}$. HAPIs have detrimental impacts on patients' physical and psychosocial wellbeing ${ }^{7,8}$. These include prolonged pain, infection, corrective surgery, social isolation and exacerbation of mental health conditions, and result in the estimated death of 60,000 patients globally ${ }^{1,6,8}$.

HAPIs also result in significant economic burden to health services worldwide ${ }^{6,9,10}$. In the United Kingdom (UK), the United States (US) and Australia, the annual cost of preventing and treating HAPls is estimated at UK $£ 5.3$ billion ${ }^{11}$, US $\$ 26.8$ billion $^{6}$ and A $\$ 985$ million respectively ${ }^{3}$. Pressure injury prevention (PIP) strategies remain at the forefront of healthcare prevention measures ${ }^{12}$, with clinical practice guidelines (CPG) providing clinicians with globally accepted best practice standards in care'.

Implementing PIP strategies include early mobilisation, specialised support surfaces, regular skin assessments, repositioning ${ }^{2,13}$, adequate nutrition, skin care, and patient education ${ }^{1,2}$. Furthermore, quality and safety initiatives aimed at reducing the burden of adverse events are emerging in healthcare, in particular programs that partner with organisations like the Institute for Healthcare Improvement $(\mathrm{IHI})^{14}$.

Care bundles are one initiative gaining traction ${ }^{15}$, providing clinicians and patients with multi-component education packages. Designed to deliver safe and effective care to targeted patients, care bundles help drive behaviour change ${ }^{16,17}$ and, if directed towards the patient, increase their participation in healthcare ${ }^{18}$. Available care bundles aim to enhance the uptake of $\mathrm{CPG}^{14}$, prevent falls ${ }^{19}$, detect sepsis $^{20}$, and prevent Pls ${ }^{15,21}$. In 2014, Australian researchers developed a patient-centred pressure injury prevention care bundle (PPIPCB) ${ }^{10,22}$ which was deemed acceptable to both patients and clinicians ${ }^{18}$.

Research shows most patients are willing participants in their PIP care $^{7}$ such as decision-making and care planning ${ }^{23-25}$. Patients who participate in their care experience improved confidence, satisfaction and clinical outcomes ${ }^{23,24,26}$. The success of implementing any patient intervention increases when nurses are also involved in the process through working in partnership with patients to achieve mutual care goals ${ }^{27,28}$.

\section{Method}

\section{Aim}

The aim of this before and after study was to implement and evaluate an evidence-informed PPIPCB ${ }^{10}$ in three acute medical units at one Australian university hospital, and to determine patients' satisfaction with the intervention (Figure 1). The research questions were:

1. Is there a difference in adult medical patients' PIP knowledge before and after their use of the PPIPCB?

2. To what extent are adult hospitalised medical patients satisfied with the PPIPCB?

\section{Design}

A quantitative intervention before and after study design, the reporting in this study followed the Standards for Quality Improvement Reporting Excellence (SQUIRE) 2.0 guidelines ${ }^{29}$.

\section{Setting}

This study was conducted across three, 28-bed acute medical units (respiratory, medical and infectious diseases) at a 750bed tertiary hospital in Queensland, Australia. The hospital has an active PIP committee that gathers and reports monthly HAPI data from all clinical units. All hospital inpatients receive the following PIP strategies: skin inspection within 8 hours of hospital admission ${ }^{1}$; Waterlow risk assessment on admission and throughout their care 30,31 ; weekly assessment of PI risk. Patients' 'usual PIP care' at the study site is individualised and can include specialised support surfaces, regular skin 
Figure 1. Study overview

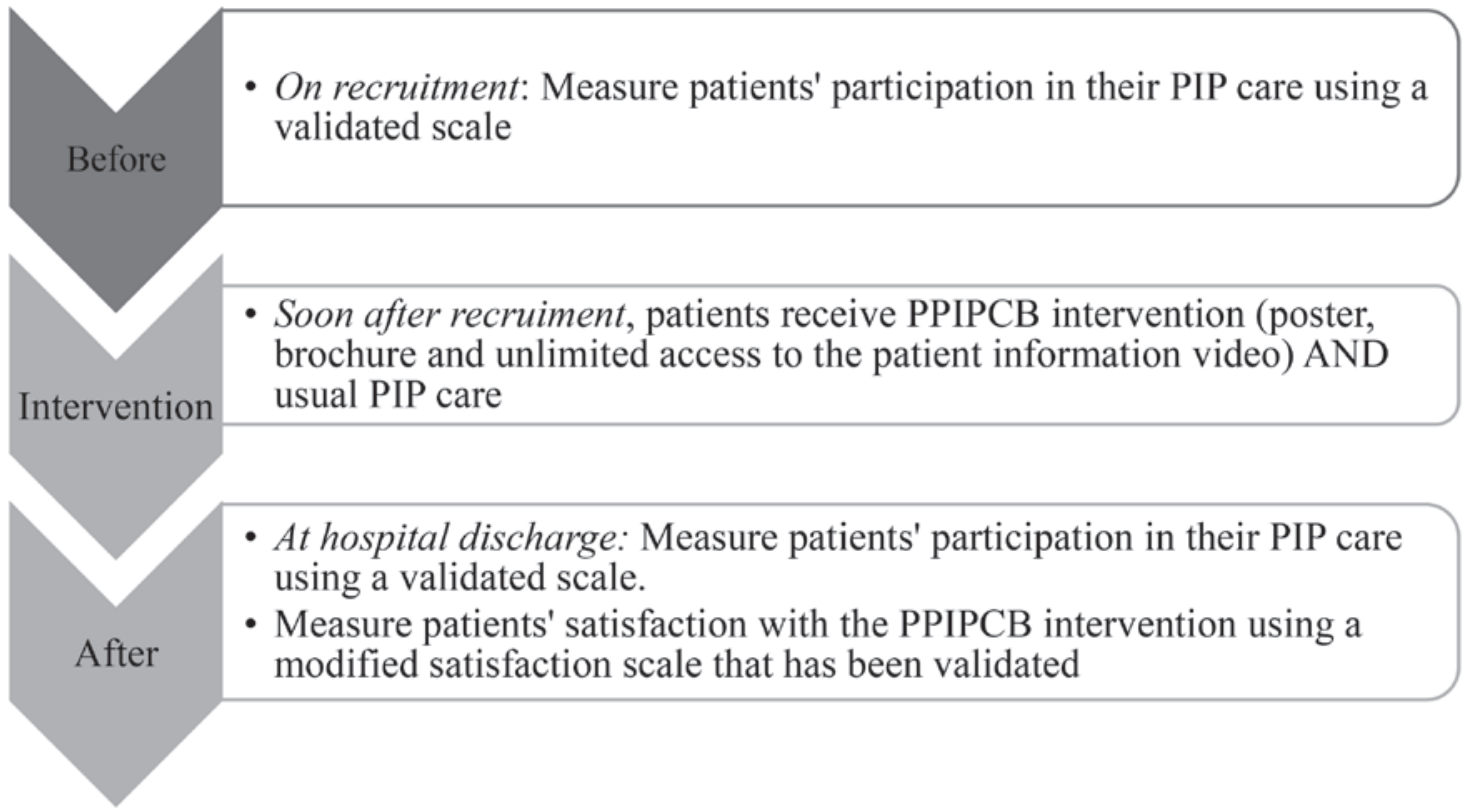

assessments, regular repositioning, adequate nutrition, skin care, and patient education ${ }^{31}$.

\section{Sample}

Potential participants were current acute inpatients in three medical units receiving their usual PIP care. From this, we recruited a consecutive sample of patients who met the following study inclusion and exclusion criteria. Inclusion criteria: $\geq 18$ years of age; able to provide written informed consent; and able to read and view the PPIPCB. Exclusion criteria: previous study recruitment; receiving palliative or end-of-life care; visual, hearing or cognitive impairment; and requiring the assistance of an interpreter. Potential participants were identified daily by the designated nurse in charge of the unit. The nurse researcher provided potential participants with a study overview, responded to their questions, and obtained written consents from willing participants.

\section{PPIPCB implementation}

This study implemented a PPIPCB developed by Australian researchers in $2014^{10}$. In addition to their usual PIP care, all recruited participants received the PPIPCB intervention comprising of a professionally developed poster, brochure and video $^{10}$ each available in nine languages - Arabic, Chinese, Croatian, English, Greek, Italian, Somalian, Spanish, Vietnamese. All resources contain three simple PIP messages and strategies for patients - "keep moving; look after your skin; and eat a well-balanced diet"10. Prior to the data collection period, the nine videos were loaded onto the patient entertainment system ${ }^{32}$, an individual interactive touch-screen bedside device that allows patients to access health information and television 24 hours a day ${ }^{32}$. The colour posters and brochures were printed for distribution to recruited participants.
The PPIPCB intervention was consistently implemented by the nurse researcher. Together, the participant and nurse researcher viewed the video, poster and brochure at the bedside. The nurse researcher then asked the participant questions regarding the information contained within the PPIPCB. All recruited participants were reminded to access the video as frequently as they wished. Using the TeachBack Method ${ }^{33}$, the nurse researcher invited participants to demonstrate their ability to access the video via the bedside technology.

\section{Data collection}

Two validated scales were used - the patient participation in pressure injury prevention (PPPIP) scale $^{34}$ and a modified multi-dimensional treatment satisfaction measure $(m M D T S M)^{35}$.

The PPPIP scale contains seven items measuring patients' PIP care participation ${ }^{34}$. It has a four-point response (1=strongly disagree to $4=$ strongly agree), with a possible total range of 7-28; higher scores indicate greater patient participation in their $\mathrm{PIP}^{34}$. The scale was shown to be valid and reliable ${ }^{34}$. The PPPIP scale was administered to participants upon study recruitment and prior to their hospital discharge (Figure 1). Participant demographics and clinical data were collected including age, gender, self-reported admission diagnosis, comorbidities, and any HAPI, both location and stage.

Patients' PPIPCB satisfaction was measured using the $m \mathrm{MDTSM}^{35}$, with permission to modify this scale sought from the researchers. The MDTSM was originally developed to measure treatment satisfaction with an insomnia intervention comprising of two domains (process, outcome), six attributes (treatment component, overall treatment, implementation, 
perceived benefits, discomfort and attribution of outcomes to treatment), 11 sub-scales and 48 items ${ }^{35}$. For our study, the MDTSM was modified to reflect satisfaction with the PPIPCB intervention ${ }^{35}$. We omitted questions pertaining to the nurse researchers' competence and interpersonal style from the original $\mathrm{scale}^{35}$ in order to minimise bias in our study as the nurse researcher administered the measure.

The mMDTSM measured patients' satisfaction with the PPIPCB intervention under the same two domains (process and outcome), but was modified to include five attributes, seven sub-scales and 18 items (Table 1). Process was measured in terms of overall treatment and implementation components, while outcome was measured in relation to perceived benefits, discomfort and attribution of outcomes to treatment. The scale uses a four-point option from $1=$ strongly disagree to $4=$ =strongly agree, with a possible range of $18-76$. Higher total scores indicated greater patient satisfaction with the PPIPCB intervention.

Data collection tools were uploaded onto a secure web-based clinical trial data management and survey tool (Research Electronic Data Capture [REDCap] ${ }^{36}$. Using a password- protected iPad which was securely logged onto the university network, participant data were input directly into REDCap. A trained nurse researcher with extensive experience in caring for medical patients collected data each weekday between 0830-1630hrs during a continuous 4-week period in November and December 2019. During data collection, the nurse researcher and chief investigator met regularly to discuss the study processes and procedures. The chief investigator randomly examined the REDCap data quality.

Following participant recruitment, the nurse researcher first gathered demographic and clinical data, then administered the PPPIP scale ${ }^{22,34}$. Next, the nurse researcher implemented the PPIPCB intervention. At hospital discharge, the PPPIP scale $^{22,34}$ and the mMDTSM scale ${ }^{35}$ were administered concurrently to participants.

\section{Ethics}

Ethical clearance was granted by the Human Research Ethics Committees of the hospital [HREC/2019/QGC/49756] and university [2019/375]. This study was guided by the 2018 updated Australian National Statement on Ethical Conduct in Human Research ${ }^{37}$.

Table 1. mMDTSM domains, attributes, sub-scales and items

\begin{tabular}{|c|c|c|c|}
\hline Domain & Attribute & Sub-scale & Items \\
\hline \multirow{3}{*}{ Process } & $\begin{array}{l}\text { PPIPCB } \\
\text { components }\end{array}$ & Utility & $\begin{array}{l}\text { 1. Useful for increasing my knowledge about PIP } \\
\text { 2. Useful for increasing my knowledge on how to prevent PIs } \\
\text { 3. Increased my confidence in participating in my PIP care }\end{array}$ \\
\hline & \multirow[t]{2}{*}{ Overall PPIPCB } & Attitude & $\begin{array}{l}\text { 1. I liked the PPIPCB } \\
\text { 2. The quality of the PPIPCB was high }\end{array}$ \\
\hline & & Engagement & $\begin{array}{l}\text { 1. The PPIPCB increased my willingness to engage with it } \\
\text { 2. The PPIPCB increased my willingness to participate in my } \\
\text { PIP care }\end{array}$ \\
\hline \multirow[t]{2}{*}{ Outcome } & Perceived benefits & Perceived benefits & $\begin{array}{l}\text { 1. The PPIPCB provided me with PIP strategies that I regularly } \\
\text { implemented during my hospitalisation } \\
\text { 2. The PPIPCB helped me to prevent sore areas on my skin } \\
\text { 3. The PPIPCB help me to talk to nurses about PIP }\end{array}$ \\
\hline & $\begin{array}{l}\text { Attribution of } \\
\text { outcomes to } \\
\text { treatment }\end{array}$ & $\begin{array}{l}\text { Attribution of } \\
\text { outcomes to } \\
\text { treatment }\end{array}$ & $\begin{array}{l}\text { 1. The PPIPCB help me to participate in my PIP care } \\
\text { 2. The PPIPCB information and strategies can be used by me } \\
\text { in other health areas }\end{array}$ \\
\hline
\end{tabular}




\section{Data analysis}

Data were analysed using IBM SPSS Statistics for Windows, Version 25.0. Prior to analysis, all data were cleaned and checked for errors. Missing data were managed by listwise deletion of the participant case for the PPPIP scale, and retained for the $m$ MDTSM where the percentage of missing data for each scale items $<15 \%{ }^{35}$. All variables were assessed for normality of distribution, skewness, kurtosis and outliers.

Descriptive analyses of the PPPIP and mMDTSM scale data were undertaken using absolute (n) and relative (\%) frequencies. Descriptive statistics (mean, standard deviation, range) were used to report on individual items on the PPPIP and $m$ MDTSM scale results. A total sum score (items 1 to 7 ) was calculated for the PPPIP scale.

A $p$ value of $<0.05$ indicated statistical significance and two-tailed tests were used ${ }^{15,35}$. For the mMDTSM scale, a total sum score - for process (items 1-13); outcome (items 14-18); and total (items 1-18) - was summed into three new satisfaction scores prior to the analysis ${ }^{35}$. Cronbach's alpha coefficient was calculated for the PPPIP and mMDTSM scale as a measure of internal consistency. Inferential analysis using a paired samples t-test were used to examine changes in the before and after PPPIP scale scores at item and total scale levels. The paired samples t-test assumptions were checked and met $^{38,39}$.

\section{Results}

\section{Demographic data}

In total, 80 participants were recruited from three medical units (respiratory: $n=32,40.0 \%$; medical: $n=28,35.0 \%$; infectious disease: $n=20,25.0 \%)$. Participants' average age was 67.2 years $(S D=18.3)$ and slightly more females $(n=42$, $52.5 \%)$ were recruited compared to males, whilst 77 out of 80 patients accessed the video in English, two in Croatian and one in Greek. In addition to the initial delivery of the intervention at recruitment, the video was watched at the bedside by $92.5 \%$ of participants (once by $n=69,86.3 \%$ and twice by $n=5,6.2 \%)$, whilst $n=6(7.5 \%)$ participants did not access the video following implementation.

PPPIP results

The mean scores for individual items on the PPPIP scale before and after the intervention are reported in Figure 2. There were statistically significant increases in total mean scores for the PPPIP scale after the intervention (before: mean=18.6, $S D=2.8$; after: mean=22.5, $S D=1.9$ ), equating to an overall effect size of $3.9(p<0.001)$. Cronbach's alpha was $<.70$ for the 7-item PPPIP scale (before: $\alpha=0.524$; after: $\alpha=0.616$ ), suggesting a low internal consistency reliability for the scale in this sample. No improvement was found for the Cronbach's alpha where individual items were deleted. The eta squared statistic (0.63) indicated a large effect size, with a substantial difference in the before and after intervention scores.

\section{mMDTSM results}

The mMDTSM scale was administered at hospital discharge. For the mMDTSM scale (Table 1), three satisfaction domain scores were derived - process, outcome and total. The mean process and outcome scores were 45.9 (range=38-52; $\mathrm{SD}=3.5$ ) and 16.5 (range=13-20; $\mathrm{SD}=2.0$ ) respectively. The total mean score was 62.2 (range=53-72; $S D=5.0$ ). The internal consistency of the summed satisfaction domain scores were: process $\alpha=.79$; outcome $\alpha=.84$; and total $\alpha=.87$,

\section{PPPIP Scale Item scores}

The pressure injury prevention care I received was right for me

Family members or friends helped me make sure my health care wishes were being followed by the nurses.

I participated in the decisions made about my pressure injury prevention care, to the extent I wanted to.

During my hospital stay, when discussions had to be made about pressure injury prevention, nurses described the good and bad things about my options.

When I wanted information about my PI care, it was easy to find a nurse to tell me what I wanted to know.

I always felt well enough to be able to talk with my nurses.

I know a lot about pressure injury risk.

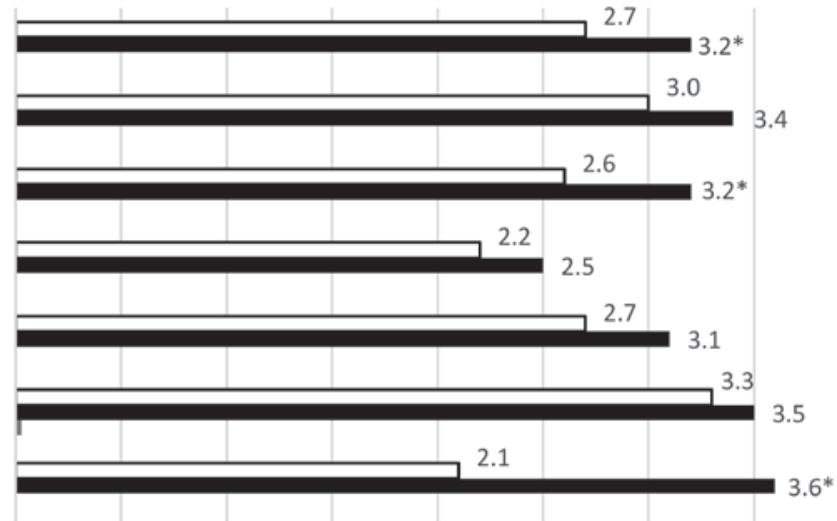

$\begin{array}{lllllllll}0 & 0.5 & 1 & 1.5 & 2 & 2.5 & 3 & 3.5 & 4\end{array}$

$\square$ Before PPIPCB Intervention a After PPIPCB Intervention

Figure 2. PPPIP scale items

* denotes $p<.0001$ 
suggesting a high level of internal consistency reliability. Overall, participants were satisfied with the intervention.

\section{Discussion}

This before and after intervention study implemented and evaluated the association between the PPIPCB intervention and adult hospital medical patients' participation in their PIP care. Additionally, patient satisfaction with the PPIPCB was measured. The PPIPCB intervention was delivered and implemented as planned, with all participants receiving the intervention in a comparable way in terms of the order of delivery - poster, brochure and then video. The inclusion criteria were broad to not restrict our sample, as the PPIPCB was designed for a wide group of patients in order to ensure our findings were generalisable to the broad in-hospital medical patient population. The singlesite small scale PPIPCB study involving 80 participants is in contrast to Chaboyer et al's. ${ }^{15}$ multi-site, parallel group cluster randomised control trial involving 1600 participants. This study builds upon existing findings, providing valuable insights into the success of the multi-component aspect of the intervention. Specifically, finding an association between the use of PIP care bundles and: improvements in participants' PIP knowledge; the perceived benefits of the PPIPCB; patients' participation in their PIP education; and participants' satisfaction with PPIPCB.

\section{Patients' PIP knowledge}

Our findings suggest an association with participants' engagement with the PPIPCB and improved PIP knowledge. In particular, the PPPIP scale item 'I know a lot about my pressure injury risk' showed a significant improvement of $42 \%$ in participants' self-reported PIP knowledge after implementation of the PPIPCB. Schoeps et al. ${ }^{40}$ also found a 14-35\% increase in patients' knowledge of PI risk in their before and after intervention study focusing on implementing a PIP patient information pamphlet across two surgical units in a Swedish University Hospital. Roberts et al. ${ }^{41}$ suggest that improved awareness of Pls motivates patients to participate in PIP. Others note that patient education information is a pre-requisite for active participation ${ }^{42}$, hence our findings align with their claim. Furthermore, Zuo and Meng ${ }^{43}$ highlight that, when care bundle components are concurrently implemented, as with the PPIPCB, a greater effect can be achieved as opposed to implementing single bundle components which may produce inferior results ${ }^{14}$. It is possible this has occurred in our study.

Whilst it is unknown which individual components of the intervention were effective, in the PPPIP scale item 'I participated in the decisions made about my pressure injury prevention care to the extent I wanted to' improved significantly after the intervention. The PPIPCB aligns with four essential components of patient participation in nursing - 'having dialogue', 'sharing knowledge', 'partaking in planning' and 'managing self-care' ${ }^{34,44}$. Additionally, we found a statistically significant improvement in patients' willingness to participate in their PIP care. Our finding differs from the original PPIPCB study conducted by Chaboyer et al. ${ }^{15}$ who found no statistical improvement at the patient level when participating in their PIP care. Increased patient participation aligns with improved health outcomes ${ }^{16,45}$, with patient participation in their healthcare encompassing engagement, collaboration, partnership, empowerment ${ }^{23,34,46}$, involvement ${ }^{46}$ and active decision-making ${ }^{47}$ in the clinical setting ${ }^{48}$.

\section{PPIPCB benefits}

Consistent with Chaboyer et al.'s ${ }^{15}$ study, our study participants retained a copy of the poster and brochure at the bedside, providing them with a quick reference to review throughout their HLOS. The PPIPCB video was accessed on more than one occasion by over $92 \%$ of participants in our study. This new and important finding might be explained by the unlimited $24 / 7$ availability of the video via bedside technology. Researchers report hospitals are increasingly using technology at the bedside, whilst some patients are self-reporting a preference for interactive learning during their HLOS ${ }^{49}$. Bedside technology provided patients with opportunities to view the video as often as desired as opposed to once only viewing via a tablet in the original study ${ }^{15}$. The creators of the PPPIP scale identified this as a limitation that may have potentially impacted their findings ${ }^{15}$. Hence, having unlimited video access to the PPIPCB at the bedside suggests that patients may benefit from a "topup dose of the intervention to reinforce messages" when increasing participation in their PIP care $^{18}$.

Because patients may have limited knowledge about PIP ${ }^{50}$, delivering health information in small multiple doses is a recognised educational approach that can empower patients to participate in their care ${ }^{23}$. Additionally, whilst only three patients accessed the video in a language other than English, the fact that it was available in multiple languages increases patient accessibility, a function not previously available to patients in the original study ${ }^{15}$. Our findings support both Roberts et al..$^{51}$ and Lavallée and colleagues ${ }^{16}$ who found health information delivered through bedside technology provides a great opportunity for hospitalised patients to engage in their PIP care, supports interactive learning opportunities, and improves patient safety in the clinical setting. Each participant at our study site had access to technology at the bedside, with the nurse researcher providing education to all participants on how to access the PPIPCB, potentially providing justification for hospitals to increase the availability of technology for patient education.

\section{Patients' participation in their PIP education}

For the PPPIP scale item 'During my hospital stay, when discussions had to be made about pressure injury prevention, nurses described the good and bad things about my options', $90 \%$ of patients disagreed with this statement before the intervention, with patients self-reporting to the nurse researcher that the intervention was the "first they had 
heard about PIP". Interestingly, Latimer et al. ${ }^{7}$ noted that patients expressed concern with a lack of PIP education conducted by nursing staff, whereby limited access to education was associated with a negative impact on PIP. Similarly, Mclnnes et al. ${ }^{19}$ identified only $37 \%$ of patients received PIP education during their HLOS. Importantly, Zingg and colleagues $^{52}$ reported healthcare workers' behaviour needs to be addressed when delivering interventions ${ }^{16}$.

Both patient education and PIP are nurse-sensitive indicators, forming two parts of Australia's national safety and quality of care Comprehensive care standard ${ }^{30}$. They are also included in the Transforming Health Care Compendium in the US ${ }^{53}$ and in the European CPGs ${ }^{1}$. Mclnnes et al. ${ }^{19}$ found PIP education provided during the early stages of their hospital stay was deemed an enabler by patients for increased participation in their PIP care. Speaking more broadly, Johnson ${ }^{54}$ states that providing nursing staff with a toolkit of patient education strategies using visual and written health resources will improve patient outcomes and aid in the delivery of safe patient care.

Hence, as a toolkit, the PPIPCB delivered multiple components in a variety of languages, incorporating pictogram images with text in both the poster and brochure, whilst audio-visual information was delivered via the patient entertainment system ${ }^{10}$. Moreover, the PPIPCB is delivered individually to the participant, increasing the potential to improve PIP knowledge and participation in PIP care ${ }^{55}$. As nurses play a vital role in patient education, having easy access to targeted evidence-based patient education resources can facilitate interpersonal dialogue between nurses and patients to enhance participation in their care ${ }^{23,25,40}$. Therefore, understanding why PIP education is not effectively delivered by nurses warrants further investigation to support the role of care bundles as a PIP strategy.

In this study, the Cronbach's alpha for the PPPIP scale was suboptimal, $\alpha<0.70$. This result is in contrast to Chaboyer et al.'s ${ }^{34}$ study which resulted in a Cronbach's alpha of 0.86 . The low Cronbach's alpha reported herein may be attributed to the small sample size of 80 participants and limited variability in participant responses, thus interpretation of results is recommended with caution. Despite this result, patients' self-reported PI knowledge improved, and an increased willingness to participate in their PIP care after the intervention was found, suggesting an association between the PPIPCB and active participation in their PIP care.

\section{Patients' PPIPCB satisfaction}

In this study, participants were satisfied with both the process and the outcome of the PPIPCB during their HLOS. According to Sidani et al. ${ }^{35}$, patient satisfaction infers a period of self-reflection and appraisal of the intervention delivered. Hence, this before and after PPIPCB intervention study also afforded patients the opportunity for a period of reflection post-implementation.
A high level of internal consistency was found in this study across all three domains (process, outcome, total), and all six attributes (treatment component, overall treatment, implementation, perceived benefits, discomfort and attribution of outcomes to treatment), with the values of Cronbach's alpha ranging from .79 to .87 , indicating reliability of the satisfaction scale. Our study correlated with Sidani et al. ${ }^{35}$ who reported Cronbach's alpha $>.70$ when validating the MDTSM in the original study.

We found $98.8 \%$ of patients were satisfied with the PPIPCB, either agreeing or strongly agreeing to the 'perceived benefits' in terms of PIP strategies that could be regularly implemented during hospitalisation: helping prevent sore areas on their skin; enabling patients to participate in their PIP care; and providing information and strategies that can be used in other health areas. Castro et al..$^{23}$ notes that patients who are satisfied with well-designed education interventions are more likely to engage with the content and adopt new behaviours in their PIP care ${ }^{50}$. Therefore, patient participation improves safety and functioning ${ }^{48,56}$ and increases confidence and satisfaction ${ }^{24,36,37}$ when compared to reduced participation rates ${ }^{45}$.

\section{Strengths and limitations}

The major strength of this before and after intervention study was the use of two validated data collection scales ${ }^{15,35}$ specific to hospitalised patients. Other strengths included that the same nurse researcher undertook all data collection. However, we acknowledge some study limitations.

First, this single-site study gathered data on participants' self-reported PIP behaviours, hence our findings cannot be generalised to other settings. Coupled with the before and after intervention study design, patients were aware that PIP knowledge was measured, which may have altered patient behaviour during the intervention period. The study could have been strengthened by having a matched control group; however, this was not possible due to the size of the hospital and resources, limiting recruiting for a second site. Second, participants were recruited on weekdays only, consequently patients were not recruited during the evening or on weekends, resulting in lower participant numbers. Therefore, this small-scale study of 80 participants limits the transferability of findings across multiple settings. However, participants were typical of the kinds of patients admitted to the hospital wards. Third, eligible patient numbers were reduced during the data collection period due to a high number of cognitively impaired patients admitted on one recruited ward. Fourth, during the study, the same nurse researcher was involved across all three phases of the intervention delivery - participant recruitment, PPIPCB intervention delivery, and administration of the before and after surveys. It is plausible that participants provided socially desirable responses as the nurse researcher had spent time to establish rapport with recruited participants. However, the nurse researcher was not involved in the 
design and development of the PPIPCB ${ }^{10}$, nor was invested in achieving positive outcomes.

Finally, because of the study design, it is always possible that other factors were responsible for the improvements in the PPPIP scores. However, to the best of our knowledge, there were no new initiatives during the data collection period that may have influenced the results. Had we been able to undertake a trial with a control group, we may have been able to rule out other factors accounting for our results.

\section{Conclusion}

This study examined the association between the delivery of the PPIPCB and increased participation and satisfaction in patients' PIP care across three medical units at a university hospital. The PPIPCB included three key messages - "keep moving; look after your skin; and eat a well-balanced diet" as part of a multi-component intervention including a poster, brochure and video delivered in this study ${ }^{10}$. We found that the PPIPCB was associated with increasing patients' selfreported knowledge of Pls and that patients were satisfied with the delivered intervention. Nursing staff reported anecdotally that the PPIPCB would assist them in educating patients in PIP. Further implementation encompassing nursing staff as interventionalists is warranted to explore the PPIPCB across various organisation contexts and populations.

The PPIPCB uptake in the wider hospital setting and across multiple sites is worth investigating. Additionally, targeted professional development training for nursing staff encompassing one-on-one mentoring, ward-based training and in-services conducted by nurse researchers trained in the PPIPCB delivery is warranted. Opportunities for engaging hospital-based PIP committees to maximise the system-wide uptake by staff may enhance accountability of practice and improve patient outcomes. This study supports the previous recommendations ${ }^{10}$ that determining nurses' perspectives is required to ascertain successful patient engagement in the PPIPCB. It is conceivable that multi-component care bundles available via bedside technology may provide increased opportunities for a wider demographic of nurses and patients to increase PIP knowledge and improve patient safety outcomes, increasing willingness for participation in their PIP ${ }^{14,34}$.

\section{Author contributions}

All authors have agreed on the final version and meet at least one of the following criteria (as recommended by the ICMJE http://www.icmje.org/recommendations/): study conceptualisation and design undertaken by SL, BG, PN, WC. Data acquisition completed by JD. Data analysis and interpretation conducted by JD, SL, BG, WC. Manuscript drafting completed by JD and critical revisions by JD, SL, WC, BG, PN. Contributions of others who did not merit authorship but participated in the research: Nil to report.

\section{Conflict of interest}

The authors declare no conflicts of interest.

\section{Funding}

The study was funded by a 2018 Sigma Theta Tau International small grant. The funders were not involved in the study design, data collection, data analysis, data interpretation nor manuscript writing.

\section{References}

1. European Pressure Ulcer Advisory Panel (EPUAP), National Pressure Injury Advisory Panel (NPIAP), and Pan Pacific Pressure Injury Alliance (PPPIA), eds. Prevention and treatment of pressure ulcers/injuries: clinical practice guidelines. 3rd ed. EPUAP, NPIAP \& PPPIA; 2019.

2. National Institute for Health and Care Excellence, editor. Pressure ulcers: prevention and management: clinical guideline. London: National Institute for Health and Care Excellence; 2014.

3. Nguyen K, Chaboyer W, Whitty J. Pressure injury in Australian public hospitals: a cost-of-illness study. Aust Health Rev 2015;39(3):329-336.

4. Li Z, et al. Global prevalence and incidence of pressure injuries in hospitalised adult patients: a systematic review and metaanalysis. Int J Nurs Stud 2020;105:103546.

5. VanGilder $\mathrm{C}$, et al. The International Pressure Ulcer Prevalence Survey: 2006-2015: A 10-year pressure injury prevalence and demographic trend analysis by care setting. J WOCN 2017;44(1):20-28.

6. Padula W, Delarmente B. The national cost of hospital-acquired pressure injuries in the United States. Int Wound J 2019;16:634640.

7. Latimer S, Chaboyer W, Gillespie B. Patient participation in pressure injury prevention: giving patients a voice. Scand $J$ Caring Sci 2014;28(4):648-656.

8. Coleman $\mathrm{S}$, et al. Patient risk factors for pressure ulcer development: systematic review. Int J Nurs Stud 2013;50(7):9741003.

9. Dealey $\mathrm{C}$, Posnett J, Walker A. The cost of pressure ulcers in the United Kingdom. J Wound Care 2012;21(6):261-266.

10. Gillespie B.M, et al. Development and pilot testing of a patientparticipatory pressure ulcer prevention care bundle. J Nurs Care Qual 2014;29(1):74-82.

11. Guest J, et al. Health economic burden that wounds impose on the National Health Service in the UK. BMJ Open 2015;5(12):e009283.

12. Australian Commission on Safety and Quality in Health Care. National safety and quality health service standards. Sydney; 2017.

13. Niederhauser $\mathrm{A}$, et al. Comprehensive programs for preventing pressure ulcers: a review of the literature. Adv Skin Wound Care 2012;25(4):167-188.

14. Institute for Healthcare Improvement $(\mathrm{IHI})$. What is a care bundle? 2014. Available from: https://www.ihi.org/resources/ Pages/ImprovementStories/WhatlsaBundle.aspx.

15. Chaboyer W, et al. The effect of a patient centred care bundle intervention on pressure ulcer incidence (INTACT): a cluster randomised trial. Int J Nurs Stud 2016;64:63-71.

16. Lavallée J.F, et al. The effects of care bundles on patient outcomes: a systematic review and meta-analysis. Implement Sci 2017;12(1):142-13. 
17. Resar R, et al. Using care bundles to improve healthcare quality. $\mathrm{IHI}$ innovation series white paper. Cambridge, MA: Institute for Healthcare Improvements; 2012.

18. Roberts S, et al. Process evaluation of a cluster-randomised trial testing a pressure ulcer prevention care bundle: a mixedmethods study. Implement Sci 2017;12(1):18.

19. Mclnnes $E$, et al. The role of patients in pressure injury prevention: a survey of acute care patients. BMC Nurs 2014;13(1):1-8.

20. Nguyen H.B, et al. Early goal-directed therapy in severe sepsis and septic shock: insights and comparisons to ProCESS, ProMISe, and ARISE. Critical Care 2016;20(1):160.

21. Downie F, Perrin A-M, Kiernan M. Implementing a pressure ulcer prevention bundle into practice. Br J Nurs 2013;22(15):S4-S10.

22. Chaboyer W, et al. INTroducing A Care bundle To prevent pressure injury (INTACT) in at-risk patients: a protocol for a cluster randomised trial. Int J Nurs Stud 2015;52(11):1659-1668.

23. Castro E.M, et al. Patient empowerment, patient participation and patient-centeredness in hospital care: a concept analysis based on a literature review. Patient Ed Counsel 2016;99(12):19231939.

24. Ledger L, et al. Patient involvement in pressure ulcer prevention and adherence to prevention strategies: an integrative review. Int J Nurs Stud 2020;101:103449.

25. Team V, et al. Patient education materials on pressure injury prevention in hospitals and health services in Victoria, Australia: availability and content analysis. Int Wound J 2020;17(2):370379.

26. Ding B, et al. Effect of patient participation on nurse and patient outcomes in inpatient healthcare. Int $\mathrm{J}$ Environ Res Public Hlth 2019;16(8):1344.

27. Tobiano G, et al. Patient participation in nursing care on medical wards: an integrative review. Int J Nurs Stud 2015;52(6):11071120.

28. Sahlsten $M$, et al. An analysis of the concept of patient participation. Nursing Forum 2008;43(1):2-11.

29. Ogrinc G, et al. SQUIRE 2.0 (Standards for QUality Improvement Reporting Excellence): revised publication guidelines from a detailed consensus process. BMJ Quality Safety 2016;25(12):986992.

30. Australian Commission for Safety and Quality in Health Care (ACSQHC). National safety and quality health service standards. Sydney: ACSQHC; 2017.

31. Queensland Health. NSQHS Standard 8 Pressure Injury - Definitions sheet 12 V3.0; 2014. Available from: https:// clinicalexcellence.qld.gov.au/sites/default/files/2017-07/pipaudit-def.pdf

32. Gold Coast Health. Patients and visitors, your time in hospital; technology: patient entertainment system (PES); 2020. Available from: https://www.goldcoast.health.qld.gov.au/patients-andvisitors/your-time-hospital/technology.

33. Centrella-Nigro A.M, Alexander C. Using the teach-back method in patient education to improve patient satisfaction. J Cont Ed Nurs 2017;48(1):47-52.

34. Chaboyer W, et al. Initial psychometric testing and validation of the patient participation in pressure injury prevention scale. $\mathrm{J}$ Adv Nurs 2017;73(9):2237-2247.

35. Sidani S, Epstein D, Fox M. Psychometric evaluation of a multi-dimensional measure of satisfaction with behavioral interventions. Res Nurs Hlth 2017;40(5):459-469.

36. Vanderbuilt University. Research Electronic Data Capture (REDCap); 2020.
37. National Health and Medical Research Council. The National statement on ethical conduct in human research. NHMRC; 2018.

38. Pallant, J. SPSS survival manual: a step by step guide to data analysis using IBM SPSS. 6th ed. Sydney, Melbourne, Auckland \& London: Allen \& Unwin; 2016.

39. Polit D, Beck C.T. Nursing research: generating and assessing evidence for nursing practice. 11th international ed. Philadelphia, PA: Wolters Kluwer/Lippincott Williams \& Wilkins; 2017.

40. Schoeps L, Tallberg A, Gunningberg L. Patients' knowledge of and participation in preventing pressure ulcers: an intervention study. Int Wound J 2017;14(2):344-348.

41. Roberts S, et al. Patients' perceptions of a pressure ulcer prevention care bundle in hospital: a qualitative descriptive study to guide evidence-based practice. Worldviews Evid Based Nurs 2017;14(5):385-393.

42. Kang $E$, et al. Discharge education delivered to general surgical patients in their management of recovery post discharge: a systematic mixed studies review. Int J Nurs Stud 2018;87:1-13.

43. Zuo X-L, Meng F-J. A care bundle for pressure ulcer treatment in intensive care units. Int J Nurs Sci 2015;2(4):340-347.

44. Eldh A.C, Luhr K, Ehnfors M. The development and initial validation of a clinical tool for patients' preferences on patient participation: the 4Ps. Health Expect 2015;18(6):2522-2535.

45. Weingart $S$, et al. Hospitalized patients' participation and its impact on quality of care and patient safety. Int J Qual Hlth Care 2011;23(3):269-277.

46. Longtin Y.M.D, et al. Patient participation: current knowledge and applicability to patient safety. Mayo Clin Proceed 2010;85(1):5362.

47. Eldh A.C, Ekman I, Ehnfors M. Conditions for patient participation and non-participation in health care. Nurs Ethic 2006;13(5):503514.

48. Phillips N.M, Street M, Haesler E. Measuring patient participation in health care: a comprehensive systematic review protocol. JBI Database System Rev Implement Reps 2014;12(3):68-88.

49. Elgin K.H, Bergero C. Technology and the bedside nurse: an exploration and review of implications for practice. Nurs Clin 2015;50(2):227-239.

50. Hartigan I, Murphy S, Hickey M. Older adults' knowledge of pressure ulcer prevention: a prospective quasi-experimental study. Int J Older People Nurs 2012;7(3):208-218.

51. Roberts S, et al. Using technology to engage hospitalised patients in their care: a realist review. BMC HIth Serv Res 2017;17(1):388-15.

52. Zingg W.M.D, et al. Hospital organisation, management, and structure for prevention of health-care-associated infection: a systematic review and expert consensus. Lancet Infect Dis 2015;15(2):212-224.

53. National Patient Safety Foundation. Transforming health care. A compendium of reports from the National Patient Safety Foundation's Lucian Leape Institute. Boston, MA: Lucian Leape Institute; 2016.

54. Johnson A. Health literacy: how nurses can make a difference. Aust J Adv Nurs 2015;33(2):21-28.

55. Yin $\mathrm{H}$, et al. Health literacy: an educationally sensitive patient outcome. J Gen Intern Med 2015;30(9):1363-1368.

56. Arnetz J.E, et al. Active patient involvement in the establishment of physical therapy goals: effects on treatment outcome and quality of care. Adv Physiother 2004;6(2):50-69. 